\title{
SOLUTIONALLY COMPLETE VARIETIES
}

\author{
HARALD HULE \\ (Received 14 November 1978; revised 14 February 1979) \\ Communicated by $\mathbf{R}$. Lidl
}

\begin{abstract}
A variety $\mathfrak{A}$ is called solutionally complete if any solvable system of algebraic equations over an algebra $A$ in $\mathfrak{A}$ which has at most one solution in every extension of $A$ in $\mathfrak{A}$ has the solution in $A$. A necessary and sufficient condition for solutional completeness is given which is a weaker form of the strong amalgamation property.
\end{abstract}

Subject classification (Amer. Math. Soc. (MOS) 1970): 08 A 15.

Let $A$ be a universal algebra in the variety $\mathfrak{A}, X=\left\{x_{1}, \ldots, x_{n}\right\}$ a finite set of indeterminates, and $W(A, X)$ the word algebra in $X$ over $A$ in the sense of Lausch and Nöbauer (1973). A system of algebraic equations (shortly 'algebraic system') in $X$ over $A$ is a family of pairs of elements of $W(A, X)$. An algebraic system $\left\langle\left(p_{i}\left(x_{1}, \ldots, x_{n}\right), q_{i}\left(x_{1}, \ldots, x_{n}\right)\right\rangle_{i \in I}\right.$ is 'solvable over $(A, \mathfrak{R})$ ' if there exist a $\mathfrak{A}$-extension $B$ of $A$ (that is, an algebra $B \in \mathfrak{I}$ which has $A$ as a subalgebra) and elements $b_{1}, \ldots, b_{n} \in B$ such that $p_{i}\left(b_{1}, \ldots, b_{n}\right)=q_{i}\left(b_{1}, \ldots, b_{n}\right)$ for all $i \in I$.

Lausch and Nöbauer (1973) posed the following problem: If $S$ is an algebraic system solvable over $(A, \mathfrak{R})$ which has at most one solution in every $\mathfrak{A}$-extension of $A$, is then the unique solution of $S$ in $A$ ?

Negative answers to this question have been given independently by Taylor (1976) and Hule (1976). In some well-known varieties, however (for instance, the varieties of groups and of lattices), the answer is affirmative. Such varieties are called 'solutionally complete'.

We want to give a necessary and sufficient condition for solutional completeness. For this purpose we consider simple extensions of an algebra $A$, that is, algebras generated by $A \cup\{b\}$ for some $b \notin A$. Such an algebra will be denoted by $A(b)$. Two simple extensions $A(b)$ and $A(c)$ will be called 'isomorphic over $A$ ' if there exists an isomorphism $\tau: A(b) \rightarrow A(c)$ such that $\tau a=a$ for every $a \in A$ and $\tau b=c$. 
THEOREM 1. A variety $\mathfrak{A}$ is solutionally complete if and only if the following condition (A) holds:

(A) For any algebra $A \in \mathfrak{A}$ and any two simple $\mathfrak{A}$-extensions $A(b), A(c)$ isomorphic over $A$ there exist $a \mathfrak{A}$-extension $D$ of $A$ and homomorphisms $\varphi: A(b) \rightarrow D$, $\psi: A(c) \rightarrow D$ such that $\varphi$ and $\psi$ fix $A$ and $\varphi b \neq \psi c$.

Proof. Suppose that $\mathfrak{A}$ satisfies condition (A). In Hule (1976) it is shown that a variety is solutionally complete if the definition is satisfied for algebraic systems in one indeterminate. So let $S=\left\langle p_{i}(x), q_{i}(x)\right\rangle_{i \in I}$ be an algebraic system in $\{x\}$ over $A$ which is solvable over $(A, \mathfrak{R})$ and has no solution in $A$. Then the system has a solution $b$ in some $\mathfrak{A}$-extension $B$ of $A$. We take the subalgebra $A(b)$ of $B$ and construct an isomorphic copy $A(c)$ of $A(b)$ by renaming the elements of $A(b)-A$ and defining an isomorphism $\tau$ such that $\tau b=c$ and $\tau a=a$ for every $a \in A$. Then $A(b)$ and $A(c)$ are simple $\mathfrak{X}$-extensions isomorphic over $A$. Let $D, \varphi, \psi$ be as in the theorem. Then $p_{i}(b)=q_{i}(b)$ implies $p_{i}(\varphi b)=q_{i}(\varphi b)$ and $p_{i}(\psi c)=q_{i}(\psi c)$ for every $i \in I$. Hence $\varphi b$ and $\psi c$ are two different solutions of $S$ in $D$. This shows that $\mathfrak{A}$ is solutionally complete.

Now suppose that condition (A) is not satisfied in $\mathfrak{A}$. Then there exist an algebra $A \in \mathfrak{A}$ and a pair of simple $\mathfrak{A}$-extensions $A(b), A(c)$ isomorphic over $A$ such that for any $\mathfrak{A}$-extension $D$ of $A$ and any pair of homomorphisms $\varphi: A(b) \rightarrow D$ and $\psi: A(c) \rightarrow D$ which fix $A, \varphi b=\psi c$. By Lemma 4.43, Chap. 1 of Lausch and Nöbauer (1973) there exist a homomorphism $\lambda$ from the polynomial algebra $A(\{x\}, \mathfrak{A})$ onto $A(b)$ with $\lambda x=b$ and $\lambda a=a$ for every $a \in A$, and a homomorphism $\mu$ from $A(\{y\}, \mathfrak{Q})$ onto $A(c)$ with $\mu y=c$ and $\mu a=a$ for every $a \in A$ (we assume $x \neq y$, without loss of generality). Let $\beta$ be the kernel of $\lambda$ and $\gamma$ the kernal of $\mu$. Now we define $D=A(\{x, y\}, \mathfrak{2}) / \delta$, where $\delta$ is the congruence on $A(\{x, y\}, \mathfrak{I})$ generated by $\beta \cup \gamma$. This means that $p(x, y) \equiv q(x, y)$ mod $\delta$ holds if and only if there exists a sequence of words $w_{0}, w_{1}, \ldots, w_{k} \in W(A,\{x, y\})$ such that $w_{0}$ is a word representation of $p(x, y), w_{k}$ represents $q(x, y)$, and for any $i \in\{1, \ldots, k\}$ either $w_{i-1}$ and $w_{i}$ represent the same polynomial or $w_{i}$ is obtained from $w_{i-1}$ replacing some subword $u$ of $w_{i-1}$ by $v$ where $u$ and $v$ represent polynomials congruent under $\beta$ or under $\gamma$. We first show that $\delta$ separates the elements of $A$. Assume $a_{1}, a_{2}$ to be different elements of $A$ and $w_{0}, w_{1}, \ldots, w_{k}$ a sequence of words as above which establishes $a_{1} \equiv a_{2} \bmod \delta$. For each $i$, let $\bar{w}_{i}$ be the element of $A(b)$ obtained from $w_{i}$ by substituting each occurrence of $x$ or $y$ by $b$ and performing the operations in $A(b)$. Then $a_{1}=\bar{w}_{0}=\bar{w}_{1}=\ldots=\bar{w}_{k}=a_{2}$. Therefore, we can consider $D$ as a $\mathfrak{U}$-extension of $A$. Since $\beta \subseteq \delta$ and $\gamma \subseteq \delta$, the canonical homomorphism $\chi$ from $A(\{x, y\}, \mathfrak{Q})$ onto $D$ induces homomorphisms $\varphi: A(b) \rightarrow D$ and $\psi: A(c) \rightarrow D$ defined by $\varphi \lambda x=\chi x, \psi \mu y=\chi y, \varphi a=\psi a=a$ for every $a \in A$. By hypothesis we have $\varphi b=\psi c$ and conclude

$$
\chi x=\varphi \lambda x=\varphi b=\psi c=\psi \mu y=\chi y,
$$


whence $x \equiv y \bmod \delta$. Now let $\left\{\left(p_{i}, q_{i}\right) i \in I\right\}$ be a generating set of the congruence $\beta$. Then the family $S=\left\langle\left(p_{i}, q_{i}\right)\right\rangle_{i \in I}$ (where we identify the polynomials $p_{i}, q_{i}$ with corresponding words) is an algebraic system over $A$ which has the solution $b$ in $A(b)$, not in $A$. We now show that $S$ has at most one solution in any $\mathfrak{A}$-extension of $A$, which will prove that $\mathfrak{A}$ is not solutionally complete. Let $e$ and $e^{\prime}$ be solutions of $S$ in some $\mathfrak{U}$-extension $E$ of $A$. Then $u(x) \equiv v(x) \bmod \beta$ implies $u(e)=v(e)$ and $u(y) \equiv v(y) \bmod \gamma$ implies $u\left(e^{\prime}\right)=v\left(e^{\prime}\right)$. Let $w_{0}, w_{1}, \ldots, w_{k}$ be a sequence of words which establishes the relation $x \equiv y \bmod \delta$. This sequence is converted into a chain of equal elements of $E$ if we substitute $x$ by $e$ and $y$ by $e^{\prime}$. This completes the proof of the theorem.

As an immediate consequence of Theorem 1 we get a sufficient condition for solutional completeness found previously by Hule (1976).

COROLLARY. A variety $\mathfrak{A}$ is solutionally complete if it satisfies the following condition (B):

(B) If $\mathrm{B}$ and $\mathrm{C}$ are $\mathfrak{A}$-extensions of an algebra $A \in \mathfrak{A}$, then there exists an algebra $D \in \mathfrak{A}$ which is a common extension of $B$ and $C$.

Condition (B) is usually called the 'strong amalgamation property'. Actually, it suffices that the strong amalgamation property hold for simple $\mathfrak{A}$-extensions of $\boldsymbol{A}$ isomorphic over $A$. A counterexample in Hule (1978) shows that this condition is not necessary for solutional completeness.

We want to prove a generalization of the preceding result for algebraic systems in arbitrary (not necessarily finite) sets of indeterminates. Thus, we consider an algebraic system $\left\langle\left(p_{i}(X), q_{i}(X)\right\rangle_{i \in I}\right.$ where $X=\left\{x_{j} \mid j \in J\right\}$ is an arbitrary set of indeterminates and $p_{i}(X), q_{i}(X)$ are elements of $W(A, X)$. The system is 'solvable over $(A, \mathfrak{2})$ ' if there exist a $\mathfrak{A}$-extension $E$ of $A$ and a family $E=\left(e_{j}\right)_{j_{E} J}$ of elements of $E$ such that $p_{i}(E)=q_{i}(E)$ for all $i \in I$. (For any $p(X) \in W(A, X), p(E)$ is the element of $E$ which we obtain by substituting each $x_{j}$ occurring in $p(X)$ by $e_{j}$ and performing the operations in $E$.)

A variety is called 'strongly solutionally complete' if the following condition holds: If $S$ is an algebraic system (in an arbitrary set of indeterminates) solvable over $(A, \mathfrak{A})$ which has at most one solution in every $\mathfrak{A}$-extension of $A$, then the unique solution of $S$ consists of elements of $A$.

In order to establish a necessary and sufficient condition for strong solutional completeness, we consider extensions $A(B)$ of $A$ where $A(B)$ is generated by $A \cup B$ and $A \cap B=\varnothing$. Two extensions $A(B)$ and $A(C)$ will be called 'isomorphic over $A$ ' if there exists an isomorphism $\tau: A(B) \rightarrow A(C)$ which takes $B$ onto $C$ and such that $\tau a=a$ for every $a \in A$. When considering extensions $A(B)$ and $A(C)$ isomorphic 
over $A$, we shall always assume that an isomorphism $\tau$ with the required properties is defined.

THEOREM 2. A variety $\mathfrak{A}$ is strongly solutionally complete if and only if the following condition (C) holds:

(C) For any algebra $A \in \mathfrak{U}$ and any two $\mathfrak{A}$-extensions $A(B), A(C)$ isomorphic over $A$ there exist a $\mathfrak{A}$-extension $D$ of $A$ and homomorphisms $\varphi: A(B) \rightarrow D$, $\psi: A(C) \rightarrow D$ such that $\varphi$ and $\psi$ fix $A$ and $\varphi b \neq \psi \tau b$ for at least one $b \in B$.

Proof. Suppose that $\mathfrak{A}$ satisfies condition (C). Let $S=\left\langle p_{i}(X), q_{i}(X)\right\rangle_{i \in I}$ be an algebraic system in $X=\left\{x_{j} \mid j \in J\right\}$ solvable over $(A, \mathfrak{R})$ which has no solution in $A$ (which means that no family $\left(a_{j}\right)_{j \in J}$ with $a_{j} \in A$ is a solution). Then $S$ has a solution $\bar{B}=\left(b_{j}\right)_{j \in J}$ in some $\mathfrak{U}$-extension $E$ of $A$ where $b_{j} \notin A$ for at least one $j$. Let $B=\left\{b_{j} \mid j \in J\right.$ and $\left.b_{j} \notin A\right\}$. Then we take the subalgebra $A(B)$ of $E$ and construct an isomorphic copy $A(C)$ of $A(B)$ by renaming the elements of $A(B)-A$ and defining an isomorphism $\tau: A(B) \rightarrow A(C)$ such that the two extensions are isomorphic over $A$. Then we define $\bar{C}=\left(c_{j}\right)_{j \in J}$ where $c_{j}=\tau b_{j}$ if $b_{j} \in B$ and $c_{j}=b_{j}$ if $b_{j} \in A$. Let $D, \varphi, \psi$ be as in condition (C), $\varphi \bar{B}=\left(\varphi b_{j}\right)_{j \in J}$ and $\psi \bar{C}=\left(\psi c_{j}\right)_{j \in J}$. Then $p_{i}(\bar{B})=q_{i}(\bar{B})$ implies $p_{i}(\varphi \bar{B})=q_{i}(\varphi \bar{B})$ and $p_{i}(\psi \bar{C})=q_{i}(\psi \bar{C})$ for every $i \in I$. Hence $\varphi \bar{B}$ and $\psi \bar{C}$ are two solutions of $S$ in $D$ which are different because $\varphi b_{j} \neq \psi \tau b_{j}=\psi c_{j}$ for at least one $b_{j} \in B$. This shows that $\mathfrak{A}$ is strongly solutionally complete.

Now suppose that condition $(C)$ is not satisfied in $\mathfrak{A}$. Then there exist an algebra $A \in \mathfrak{A}$ and a pair of $\mathfrak{Q}$-extensions $A(B), A(C)$ isomorphic over $A$ such that for any $\mathfrak{U}$-extension $D$ of $A$ and any pair of homomorphisms $\varphi: A(B) \rightarrow D$ and $\psi: A(C) \rightarrow D$ which fix $A, \varphi b=\psi \tau b$ for every $b \in B$. We index $B$ and $C$ by an appropriate set $J$ such that $B=\left\{b_{j} \mid j \in J\right\}, C=\left\{c_{j} \mid j \in J\right\}$ and $c_{j}=\tau b_{j}$ for all $j \in J$, then we take disjoint sets of indeterminates $X=\left\{x_{j} \mid j \in J\right\}$ and $Y=\left\{y_{j} \mid j \in J\right\}$. There exist a homomorphism $\lambda$ from $A(X, \mathfrak{A})$ onto $A(B)$ with $\lambda x_{j}=b_{j}$ for every $j \in J$ and $\lambda a=a$ for every $a \in A$, and a homomorphism $\mu$ from $A(Y, \mathfrak{Q})$ onto $A(C)$ with $\mu y_{j}=c_{j}$ for $j \in J$ and $\mu a=a$ for $a \in A$. Let $\beta$ be the kernel of $\lambda, \gamma$ the kernel of $\mu$, and $D=A(X \cup Y, \mathfrak{A}) / \delta$, where $\delta$ is the congruence on $A(X \cup Y, \mathfrak{A})$ generated by $\beta \cup \gamma$. Like in the proof of Theorem 1, taking for $\bar{w}_{i}$ the element of $A(B)$ obtained by substituting each $x_{j}$ or $y_{j}$ occurring in $w_{i}$ by $b_{j}$, we see that $\delta$ separates $A$, and hence we consider $D$ as an extension of $A$. Homomorphisms $\varphi: A(B) \rightarrow D$ and $\psi: A(C) \rightarrow D$ are defined by the conditions $\varphi \lambda x_{j}=\chi x_{j}, \psi \mu y_{j}=\chi y_{j}$ and $\varphi a=\psi a=a$ for every $a \in A$, where $\chi$ is the canonical homomorphism from $A(X \cup Y, \mathfrak{R})$ onto $D$. By hypothesis we have $\varphi b_{j}=\psi \tau b_{j}=\psi c_{j}$ for every $j$ which implies $x_{j} \equiv y_{j} \bmod \delta$. Also the rest of the proof is analogous to that of Theorem 1 . The algebraic system $S$ constructed as in that proof has the solution $\bar{B}=\left(b_{j}\right)_{j \in J}$ in $A(B)$, not in $A$, and for two arbitrary solutions of $S$ in some $\mathfrak{A}$-extension of $A,\left(e_{j}\right)_{j \in J}$ and $\left(e_{j}^{\prime}\right)_{j \in J}$, we deduce $e_{j}=e_{j}^{\prime}$ from $x_{j} \equiv y_{j} \bmod \delta$. So $\mathfrak{A}$ is not strongly solutionally complete. 


\section{References}

H. Hule (1976), 'UUber die Eindeutigkeit der Lösungen algebraischer Gleichungssysteme', J. Reine Angew. Math. 282, 157-161.

H. Hule (1978), 'Relations between the amalgamation property and algebraic equations', J. Austral. Math. Soc. Ser. A 25, 257-263.

H. Lausch and W. Nöbauer (1973), Algebra of polynomials (North-Holland, Amsterdam).

W. Taylor (1976), 'Pure compactifications in quasi-primal varieties', Canad. J. Math. 28, 50-62.

Departamento de Matemática

Universidade de Brasília

Brasilia

Brazil 